\title{
Effectivity Rts And Gap To Predict Mortality Patients Of Head Injury At Emergency Ward Of Ulin Hospital Banjarmasin
}

Subhannur Rahman ${ }^{1 *}$

${ }^{1}$ Department of Science Nursing, STIKES Sari Mulia, Banjarmasin Indonesia subhannur@stikessarimulia.ac.id

Rina Al Kahfi ${ }^{1}$

${ }^{1}$ Department of Science Nursing, STIKES Sari Mulia, Banjarmasin Indonesia Rina_alkahfi@stikessarimulia.ac.id

Bagus Rahmat Santoso ${ }^{1}$

${ }^{1}$ Department of Science Nursing, STIKES Sari Mulia, Banjarmasin Indonesia Bagus_rahmat_santoso@stikessarimulia.ac.id

Dian Kustanti ${ }^{1}$

${ }^{1}$ Department of Science Nursing, STIKES Sari Mulia, Banjarmasin Indonesia diankustanti@gmail.com

\begin{abstract}
Objective: The purpose of this study to know affectivity of RTS (Revised Trauma Score) and GAP (GCS, Age, Blood Systolic Pressure) to predict mortality patients of head injury at Emergency Ward in Ulin Hospita Banjarmasin

Method: The methods in the study were cross-sectional. The data were taken from a medical record from 1 January - 31 March 2016. Data collected based on the results of trauma scoring. Outcomes (died and life) recorded in the first 24 hours since the patient has entered the hospital. The data were analyzed by using chi-square test.

Results : 109 patients of head injury came to the emergency room of Ulin Hospital Banjarmasin approximately 36, 5 years old and 11 patients were died (10\%). Prediction mortality with scoring RTS $\mathrm{p}=0.000, \mathrm{OR}=2.390$, probabilities $=70.5 \%(95 \% \mathrm{CI}=1.893-$ 3.019). While a prediction with scoring GAP $\mathrm{p}=0.000, \mathrm{OR}=41.550$, probability $=97.6 \%$ $(95 \% \mathrm{CI}=5.589-308.876)$.

Conclusion: Scoring GAP was more effective than RTS to predict mortality patients of a head injury in the emergency ward within 24 hours with probabilities about $97.6 \%$ compared to the RTS that had a about $70.5 \%$ probability.
\end{abstract}

Keywords: GAP, Head Injury, Prediction of Mortality and RTS

\section{INTRODUCTION}

The injury scoring system tries to determine the severity to number, helping to quantitatively assess the severity of head injury [1]. The injury is a sensitive condition. Particularly during the first hour after a trauma, assessment, resuscitation, and treatment measures are the most important.

The initial handling of injured patients at the center of the trauma may decrease the patient's mortality rate. Trauma scoring assists the assessment into the correct diagnosis by 
providing a high degree of sensitivity, as the most important factor in deciding the severity of the injured patient [1]. Traumatic scoring can be used in two situations where the patient before arriving at the hospital to determine the need for referral or may also be used by emergency ward health workers when meeting patients who come with head injuries to prepare for surgery, call in the surgeon and inform the family of the severity of the conditioned patient [2].

Monitoring of head injury patients with repeated and systematic assessment may identify early improvements or deterioration of the patient. Methods that can be used in predicting improvement or deterioration in head-injured patients with RTS and GAP [1,2]. RTS (Revised Trauma Score) was introduced the system, most widely used as a physiological assessment system with high reactivity in predicting the accuracy of death and is so frequently used around the world. The system combines the values of Glasgow Coma Scale (GCS), respiratory rate and systolic blood pressure [3]. In 2011 found the latest trauma system by combining Glasgow Coma Scale assessment, age and systolic blood pressure (GAP). In this GAP scoring, there are several similarities with RTS, ie equations in the GCS scoring system and systolic blood pressure checks. While the difference is if the RTS there is a respiratory frequency assessment but the GAP using age criteria [1]. Based on a preliminary study at H. Moch. Ansari Saleh Hospital obtained data that during January to June 2015 patients with CKR 31 patients, CKS 6 patients, CKB 5 patients and 1 patient died. While in Ulin Hospital Banjarmasin there were patients with CKR 372 patients, CKS 100 patients, and CKB 106 patients. The aim of the study was to determine the effectiveness of RTS with GAP in predicting mortality in head injury patients in the emergency room.

\section{RESEARCH METHOD}

The location of the research at the emergency ward of Ulin Hospital Banjarmasin and the emergency ward of $\mathrm{H}$. Moch. Ansari Saleh hospital Banjarmasin. The population in this study was 620 patients, representing the number of all head injuries patients (578 people) in the emergency ward at Ulin Hospital in 2015 amounted to 578 patients and emergency room of $\mathrm{H}$. Moch. Ansari Saleh Hospital were 42 patients. The method was cross-sectional. The technique used in sampling was accidental sampling technique. In this case, the sample was a head injury patient the emergency room Ulin Hospital Banjarmasin with criteria samples aged 15-75 years and male and female sex and not in the influence of alcoholic beverages. Sampling was conducted on January 1 - March 31, 2016. The statistical test used by the researchers is a chi-square test using computerized. 


\section{RESULTS}

\section{A. Univariate Analysis}

The sample in this study was head injury patients (64 or 58.7\%) and at emergency ward of H. Moch. Ansari Saleh Hospital 45 patients (41.3\%). Based on the research results obtained data as follows:

Table 1. Demographic and mortality data in head injury patients Variable $\mathrm{n}(\%)$

\begin{tabular}{llc}
\hline \multicolumn{2}{c}{ Variable } & $\mathrm{n}(\%)$ \\
\hline Gender & Man & $67(61 \%)$ \\
Wge & Women & $42(39 \%)$ \\
& $<60$ Years & \\
$\geq 60$ Years & $95(87 \%)$ \\
Average Age (mean), the year of & $14(13 \%)$ \\
Diagnose while in the & $36.5(15-75)$ \\
Emergency Room & \\
CKR & CKS & $57(53 \%)$ \\
CKB & $32(29 \%)$ \\
Mortality Rate & $20(18 \%)$ \\
\multicolumn{2}{c}{ Died } & $11(10 \%)$ \\
Life & $98(90 \%)$ \\
Total Cases & & 109
\end{tabular}

Based on table 1. it could be seen that from 109 patients, 67 patients (61\%) were male and 42 patients (39\%) were female. The average age of patients with head injury was aged 36.5 years (15 - 75 years range). Upon arrival at the initial admissions diagnosis, patients with CKR were 57 patients (53\%), CKS patients were 32 patients (29\%), and CKB patients were 20 patients (18\%). While the mortality rate of patients died were 11 patients (10\%) and alived was 98 patients $(90 \%)$.
Table 2. Classification of RTS and GAP

\begin{tabular}{ll}
\hline Score RTS & \multicolumn{1}{c}{ Score GAP } \\
\hline Systolic Blood Pressure (mmHg) & $\begin{array}{l}\text { Systolic Blood Pressure } \\
(\mathrm{mmHg})\end{array}$ \\
& $\begin{array}{l}\mathrm{BP}>120 \mathrm{mmHg}=6 \\
\mathrm{BP} 60-120 \mathrm{mmHg}=4 \\
\mathrm{BP}<60 \mathrm{mmHg}=0\end{array}$ \\
& $\begin{array}{l}\text { Age } \\
\text { Age }<60 \text { Years }=3\end{array}$ \\
\hline Frequency of Breath (Minutes) & Glasgow Coma Scale \\
& Value of GCS $=3-15$ \\
\hline Glasgow Coma Scale & GAP $=$ Age + BP of \\
Value of GCS $=3-15$ & \multicolumn{1}{c}{ systolik +} \\
RTS $=\left(0.9368^{*}\right.$ Value of GCS) + & Value of GCS \\
$\left(0.7326^{*}\right.$ BP systolic value $)+$ & Skor: \\
$\left(0.2908^{*}\right.$ Respiratory Frequency) & High risk $=3-10$ \\
\hline Score: & Medium risk $=11-18$ \\
High risk $=<3.4$ & Low risk $=19-24$ \\
Medium risk $=3,4-7,2$ & \\
Low risk $=>7,2$ & \\
\hline RTS $=$ Revise trauma score; GAP $=$ Glasgow Coma Scale, age, \\
and arterial pressure
\end{tabular}

Table 2 was a classification table in the calculation of trauma scores of RTS and GAP, as well as determining risk levels based on the results of both RTS and GAP scores.

Table 3. Patient mortality rates for 24 hours after arrival at the Emergency Room

\begin{tabular}{llll}
\hline \multicolumn{1}{c}{ Variable } & \multicolumn{1}{c}{$\begin{array}{l}\text { Dead } \\
\mathrm{n}(\%)\end{array}$} & \multicolumn{1}{c}{$\begin{array}{c}\text { Life } \\
\mathrm{n}(\%)\end{array}$} & $\begin{array}{l}\text { Total } \\
\mathrm{N}\end{array}$ \\
\hline $\begin{array}{l}\text { RTS } \\
<3.4 \text { (High risk) }\end{array}$ & $0(0 \%)$ & )$(0 \%)$ & 0 \\
$\begin{array}{l}3.4-7.2 \text { (Medium risk) } \\
>7.2 \text { (Low risk) }\end{array}$ & $11(10 \%)$ & $71(38 \%)$ & 52 \\
$\quad 0(0 \%)$ & $57(52 \%)$ & 57 \\
Total & $11(10 \%)$ & $78(90 \%)$ & 109 \\
$3-10$ (High risk) & $5(4,5 \%)$ & $1(0,9 \%)$ & 6 \\
$11-18$ (Medium risk) & $6(5,5 \%)$ & $18(16,6 \%)$ & 24 \\
$19-24$ (Low risk) & $0(0 \%)$ & $79(72,5 \%)$ & 79 \\
$\quad$ Total & $11(10 \%)$ & $78(90 \%)$ & 109
\end{tabular}

Based on 3 it showed that the results of the RTS and GAP trauma measurement to determine the patient's mortality rate for 24 hours after arriving at the ER (emergency ward). Based on RTS scores with RTS <3.4 (high risk) there were no patients alive or dead. RTS 3.4 - 7.2 (moderate risk) patients were 11 dead (10\%) and 41 living patients (38\%). RTS value> 7.2 (low risk) patients get as much alive 57 (52\%) and no patients died. While on GAP score with GAP value 3 - 10 (high risk) got 5 patients died (4.5\%) and 1 patient live (0.9\%). GAP 11 - 18 (medium risk) patients were found dead as many as 6 
patients $(5.5 \%)$ and living patients as many as 18 patients (16.6\%). GAP 19 - 24 (low risk) patients were alive as much as 79 patients $(72.5 \%)$ and no patients died.

\section{B. Bivariate Analysis}

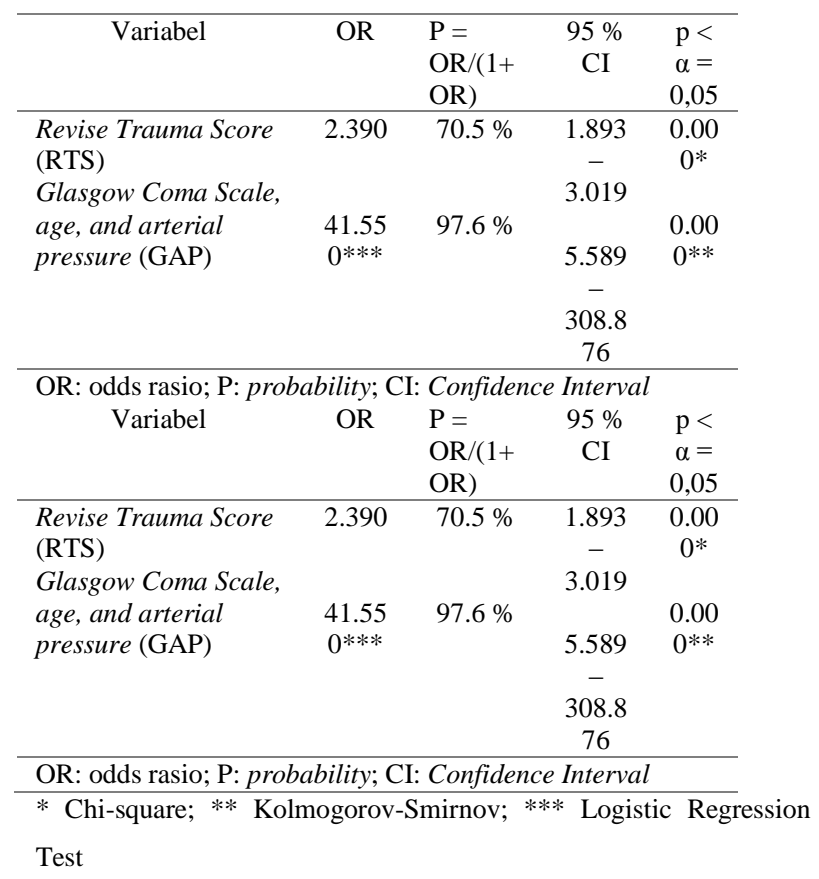

In the RTS scores when tested using chi-square test, the test results showed the value of $p=0.000, p<\alpha=0.05$, the value of $\mathrm{OR}=2,390$, $($ with $95 \%$ confidence $=1.893$ 3.019), $\mathrm{P}=70.5 \%$, it could be expressed the RTS scores were effective in predicting the mortality of head injury patients in the ER within 24 hours with a probability value of $70.5 \%$. While on GAP score based on Kolmogorov - Smirnov test obtained significance value 0.000 . Therefore, the value of $p<\alpha=0.05$, it could be concluded that GAP scoring was effective in predicting patient mortality for 24 hours. In the logistic regression test results showed significant value 0.000 , OR value: 41.550 , then the probability value is $\mathrm{P}=(\mathrm{OR} / 1+\mathrm{OR})$ then obtained the result $97.6 \%$, with $95 \%$ CI value was 5.589 - 308.876 .

\section{DISCUSSION}

1. The incidence of head injury patients at emergency room Ulin Hospital Banjarmasin

The incidence of head injury for 3 months starting from January 1 to March 31, 2016, there were 109 patients who suffered head injuries and brought to Ulin Hospital Banjarmasin, in the Emergency ward Ulin Hospital were 64 patients $(58.7 \%)$ and the emergency ward of. H. Moch. Ansari Saleh Hospital was 45 patients (41.3\%). The patient criteria were patients with CKR of 57 patients (53\%), CKS patients (32 patients (29\%), and CKB patients 20 patients (18\%).

2. RTS scoring in predicting mortality of head injured patient at the emergency room of Ulin Hospital Banjarmasin.

RTS scoring was the most widely used scoring in the patient's physiological assessment because it had a high-reliability value in predicting mortality. The score assessed the patient's preliminary assessment of GCS values, respiratory rate and systolic blood pressure [4]. The RTS scoring was a scoring that assesses the psychological system in general, with a very high value of interrater reliability and showed accurately prediction of death. RTS scoring was useful in determining quality assurance and predicting outcomes and scores more 
specifically used for patients with head injury. Score on the RTS score was $0-7.8404$. The value was useful for prehospital triage, outcome evaluation, and quality assurance in critical service [3]. RTS scoring had some limitations that affected its usefulness. The limitation was related to GCS measurement. GCS was used in measuring the functional status of the central nervous system. In the head injury was important to determine the outcome of trauma in patients. In the RTS component, the GCS had limitations in scoring measurements on intubated patients and assisted by ventilators [5,6]. Determination of verbal response and respiratory rate was very difficult to do on the condition of the patient. In addition, patients who are under the influence of alcoholic beverages or drugs and drug abuse affecting the central nervous system will also have difficulties in scoring assessments as well as mentally ill patients $[5,6]$. In this study, when the RTS scores were tested using chi-square test, $\mathrm{p}=0.000, \mathrm{p}<\alpha=0.05, \mathrm{OR}=2,390$, $(95 \%$ confidence $=1.893-3.019), \mathrm{P}=70.5 \%$ it was stated that the RTS scores were effective in predicting the mortality of head injury patients in the ER within 24 hours with a probability value of $70.5 \%$.

3. GAP scoring in predicting mortality of head injury patients at emergency room Ulin Hospital Banjarmasin

GAP Scoring is the latest MGAP modified trauma assessment system [1], In 2011, which is claimed to predict mortality in trauma patients, better than previous scores, simpler scoring. The Scoring system trauma calculates the initial assessment of patients consisting of Glasgow Coma Scale (GCS), age and systolic blood pressure [1,7]. GAP scoring has some limitations similar to RTS. The limitation is related to GCS measurement. GCS is used in measuring the functional status of the central nervous system. In head injuries, it is important to determine the outcome of trauma in patients [5,6]. In this study, when the GAP scoring test using the Kolmogorov - Smirnov test showed $\mathrm{p}=0.000, \mathrm{p}<\alpha=0.05$, the logistic regression test $\mathrm{OR}=41.550,(95 \%$ confidence $=5.589$ $308.876), \mathrm{P}=97.6 \%$, it could be stated that GAP scoring was effective in predicting mortality of head injury patients in the ER within 24 hours with a probability value of $97.6 \%$.

4. The effectiveness of RTS and GAP in predicting mortality of head injury patients at emergency department of Ulin Hospital Banjarmasin

RTS scoring is a score that assesses the patient's initial assessment of calculation of GCS values, systolic blood pressure, and respiratory rate. GAP scoring has similarities to the RTS scores in calculating the patient's initial assessment of GCS, systolic blood pressure and age. The difference is in GAP scoring using age scoring while on RTS scoring using the respiration rate assessment [1]. The GCS assessment has a large association between low GCS scores and poor 
outcomes [8]. In the study, 11 patients $(10 \%)$ died with GCS score $\leq 7$. Patients who experienced elevated systolic blood pressure $\geq 140$ were 29 patients $(26.6 \%)$, in systolic blood pressure $\geq 140$ no patients died. While the patient which had hyperventilation, respiration $>22 \mathrm{x} / \mathrm{min}$ as many as 7 patients (6.4\%) who died and 56 patients $(51.4 \%)$ was alive. Hypotension can lead to an increase in ICT to be high and allow patients for hypovolemic shock. If this continues the patient will experience worsening vital signs including respiration and death. Based on the study the number of patients with blood pressure $\leq 90$ as many as 3 patients $(2.75 \%)$ who died and respiration $<16 \times$ / minute as many as 3 patients $(2.75 \%)$ who died. The average age of most, in this case, was 36.5 years (age 15 - 75 years). This can be explained because the age range was included in the range of productive age, active working age, high mobility rate, high number of traffic accidents and the level of violence $[9,10]$. Old age has an effect in worsening outcomes after a patient has experienced trauma and is a risk factor for a poor prognosis during hospitalization [11,12]. In calculating the GAP score there is an age rating, if the patient age is $<60$ years then the value is 3 whereas $>$ 60 years the value is 0 [1]. Based on research conducted by researchers, age patients $>60$ years as many as 3 patients $(23 \%)$ who died and 10 patients (77\%) were alive. A total of 8 patients $(8.3 \%)$ who died with an age range of $15-45$ years and 88 patients $(91.7 \%)$ lived $<60$ years of age. It proved the most productive age with a head injury and age $<60$ years has a low prognosis of $8.3 \%$ compared to age $>60$ years ie $23 \%$. In the RTS scores when tested using chi square test, obtained $\mathrm{p}$ value $=$ $0.000, \mathrm{p}<\alpha=0.05, \quad \mathrm{OR}=2,390, \quad(95 \%$ confidence $=1.893-3.019), \mathrm{P}=70.5 \%$, it could be stated that the RTS score was effective in predicting the mortality of head injury patients in the ER within 24 hours with a probability value of $70.5 \%$. While the test results Kolmogorov - Smirnov to test the GAP score shows the value $\mathrm{p}=0.000, \mathrm{p}<\alpha=$ 0.05 , then to determine the value of OR using the Logistic Regression test with the result value $\mathrm{OR}=41.550,(95 \%$ confidence $=5.589$ - 308.876), $P=97.6 \%$, it was stated that GAP scoring was effective in predicting mortality of head injury patient in ER space within 24 hours with probability value $97.6 \%$. So from both scores GAP scoring conclusions were found to be more effective than RTS in predicting mortality of head injury patients in the ER within 24 hours with a probability value of $97.6 \%$. This is in line with [1] which states that GAP scoring can predict mortality more accurately than previous trauma scores: MGAP, RTS, T-RTS and TRISS. Also in line with the research [13] which states in patients with major trauma, GAP scoring is very easy to use when patients arrive in the ER so as to determine appropriate action and predict patient mortality. But this study is not in line with research conducted by [2] which states there is no significant difference in the $\mathrm{T}$ - 
RTS, MGAP and GAP scoring system in predicting the mortality of trauma patients entering the emergency room of RSCM resuscitation room throughout 2011. The results of this study are expected to be the primary and secondary data material in subsequent research. The researchers further investigated the problem of other factors that could affect the patient's mortality rate other than the trauma score computation component (GCS, age, systolic blood pressure and respiration). In addition, what a step if the next study does not take patient samples with referrals, then restrictions on patients with GCS $<13$ and patient follow-up time more than 24 hours so it can be known to deteriorate or repair patients, so the mortality rate obtained more and the better.

\section{Conclusion}

Total cases of head injury patients as many as 109 patients, consisted of 64 (58.7\%)from emergency ward of Ulin Hospita and 64 (58\%) from H. Moch. Ansari Hospital. Scoring of RTS is effective in predicting mortality of head injury patients at emergency room at Ulin Hospital Banjarmasin within 24 hours with probability value of $70.5 \%$

GAP scoring is effective in predicting the mortality of head injury patients at the emergency room of Ulin Hospital Banjarmasin within 24 hours with a probability value of $97.6 \%$

The GAP scoring system is more effective at the probability level of $97.6 \%$ compared with the RTS score of $70.5 \%$ in predicting mortality in head injury patients in the emergency room at Ulin Hospital Banjarmasin.

\section{REFERENCES}

[1] Kondo, Y., Abe, T., Kohshi, K., Tokuda, Y., Cook, EF. \& Kukita, I. 2011. Revised Trauma Scoring System to Predict inHospital Mortality in The Emergency Department: Glasgow Coma Scale, Age, and Systolic Blood Pressure Score. Kondo et al.; licensee BioMed Central Ltd.

[2]. Tirtayasa, Pande M.W. dan Philippi, Benny. 2013. Prediction of Mortality Rate of Trauma Patients in Emergency Room at Cipto Mangunkusumo Hospital by Several Scoring Systems. Med J Indones. 2013;22:227-31. doi: 10.13181/mji.v22i4.603

[3]. Champion HR, Sacco WJ, Copes WS, Gann DS, Gennarelli TA, Flanagan ME. A revision of the Trauma Score. $\mathrm{J}$ Trauma. 1989;29:623-629. doi: 10.1097/00005373-198905000-00017

[4]. Raum MR, Nijsten MW, Vogelzang M, et al, for the Polytrauma Study Group of the German Trauma Society. Emergency trauma score: an instrument for early estimation of trauma severity. Crit Care Med. 2009 Jun. 37(6):1972-7.

[5]. Chawda MN, Hildebrand F, Pape HC, Giannoudis PV. Predicting outcome after multiple trauma: which scoring system? Injury. 2004 Apr : 35(4):347-58. 
[6]. Yates DW. ABC of major trauma. Scoring systems for trauma. BMJ. 1990 Nov 10:301(6760):1090-4.

[7]. Hasler, RM., Exadaktylos, AK., Mealing, N., Juni, P. \& Lecky, F. 2012. Validation of two new Trauma Scores: MGAP and GAP. International Conference of Medicine 2012 DUBLIN, INSELSPITAL Universitatsspital Bern Hospital Universitaire De Berne Bern University Hospital.

[8]. Salama, DI., Mary, AG. \& Hamed, W. 2015. Identification of Clinical and Radiological Predictors of Outcome in Head Trauma Patients in the Emergency Department. Biolife, 3(3), pp 644-652. doi:10.17812/blj.2015.3311 Biolife 2015 | Vol 3 | Issue 3

[9]. Burns, J.Jr dan Hauser, WA. 2007. The Epidemiology Of Traumatic Brain Injury: A Review. Epilepsia.

[10]. Tasmono, H. 2011. Head Trauma On Motorcycle Accident. Saintika Medica.

[11]. Salama, DI., Maray, AG. \& Hamed, W. 2015. Identification of Clinical and Radiological Predictors of Outcome in Head Trauma Patients in the Emergency Department. Biolife, 3(3), pp 644-652. doi:10.17812/blj.2015.3311 Biolife 2015 | Vol 3 | Issue 3

[12]. Ayaz, T., Sahin, SB., Sahin, OZ., Bilir, O. \& Rakici, H. 2014. Factors Affecting Mortality in Elderly Patients Hospitalized for Nonmalignant Reasons. Journal of Aging Research
Volume 2014, Article ID 584315, 7 pages

http://dx.doi.org/10.1155/2014/584315

[13]. Ahun, E., Koksal, O., Sugar, D., Torun, G., Donmez, SS. \& Armagan, E. 2014. Value of The Glasgow Coma Scale, Age, and Arterial Blood Pressure Score for Predicting the Mortality of Major Trauma Patients Presenting to The Emergency Department. Ulus Travma Acil Cerrahi Derg, July 2014, Vol. 20, No. 4 